\title{
Validation of Malay version of Montreal cognitive assessment in patients with cognitive impairment
}

\author{
Wee Kooi Cheah ${ }^{1}$, Hoon Lang Teh ${ }^{2}$, Diana Xiao Han Huang ${ }^{3}$, Alan Swee Hock Ch'ng ${ }^{4}$, \\ Mun Pung Choy ${ }^{5}$, Ewe Eow Teh ${ }^{6}$, Irene Looi ${ }^{4}$ \\ ${ }^{1}$ Medical Department, Hospital Taiping, Perak, Malaysia \\ ${ }^{2}$ Medical Department, Hospital Kulim, Kedah, Malaysia \\ ${ }^{3}$ Clinical Research Center, Hospital Pulau Pinang, Penang, Malaysia \\ ${ }^{4}$ Medical Department, Hospital Seberang Jaya, Penang, Malaysia \\ ${ }^{5}$ Clinical Research Center, Hospital Taiping, Perak, Malaysia \\ ${ }^{6}$ Psychiatry Department, Hospital Pulau Pinang, Penang, Malaysia
}

\section{Email address:}

wkcheah@hotmail.com (W. K. Cheah), hoonlang@yahoo.com (H. L. Teh), dianahxh@gmail.com (D. X. H. Huang), alanchng@yahoo.com (A. S. H. Ch’ng), choymp@crc.gov.my (M. P. Choy), eeteh2000@yahoo.com (E. E. Teh), irenelooi@yahoo.com (I. Looi)

\section{To cite this article:}

Wee Kooi Cheah, Hoon Lang Teh, Diana Xiao Han Huang, Alan Swee Hock Ch'ng, Mun Pung Choy, Ewe Eow Teh, Irene Looi. Validation of Malay Version of Montreal Cognitive Assessment in Patients with Cognitive Impairment. Clinical Medicine Research.

Vol. 3, No. 3, 2014, pp. 56-60. doi: 10.11648/j.cmr.20140303.11

\begin{abstract}
Background: Montreal Cognitive Assessment (MoCA) has been shown to be a sensitive tool for cognitive assessment. There are high proportion of Malaysian elderly with limited proficiency in English language. Malay language is a more familiar language across the multiracial population of Malaysia. Objective: The aim of this study is to validate the Malay version of Montreal Cognitive Assessment (MMoCA) in cognitive impairment patients. Methods: Elderly aged 60 years and above were recruited by using convenient sampling method from 4 government hospitals. Subjects were categorized into normal control group versus patients group with cognitive impairment (Alzheimer's Disease (AD) and Mild Cognitive Impairment (MCI)). All subjects completed MMoCA \& MMSE - Malay version, followed by a second assessment, which involved Clinical Dementia Rating (CDR), clinical neurological and psychiatry assessment. Results: Total of 66 subjects was enrolled in the study, 44 were normal control, 14 with AD, 8 with MCI. MMoCA is better than MMSE-Malay in differentiating CDR 0 from CDR $>0$. With the cut off point of less than 22, MMoCA has the sensitivity of 0.824 and specificity of 0.818 to detect cognitive impairment. Whereas MMSE-Malay only has sensitivity of 0.765 and specificity of 0.636 with the cut off point of less than 27. Conclusion: The MMoCA is a validated and useful cognitive screening instrument in patients with cognitive impairment.
\end{abstract}

Keywords: MMSE - Malay: Malay version of Mini Mental State Examination,

MMoCA: Malay version of Montreal Cognitive Assessment, CDR: Clinical Dementia Rating,

AD: Alzheimer's Disease, MCI: Mild Cognitive Impairment

\section{Introduction}

More than 64 million of people living in Asia-Pacific region are estimated to suffer from dementia by the year 2050 [1]. Malaysia is a nation with its population undergoing aging process. In 2010, Malaysia has 2.3 million elderly people, as defined as age 60 years and above, compared to 1.5 million in the year 2000 [2]. As the incidence of dementia increases with age, the number of people living with dementia will increase as well.
In Malaysia, the Malays constitute the majority of the population (63.1\%) in the Peninsular Malaysia [2]. For them, the Malay language is their first language. In addition, even for the other races, the ability to speak Malay is contributed to the fact that Malay language has been adopted as the national language since its independence in 1957.

According to the Human Development Report by UNDP 2011 , current adult literacy rate of our population is $92.5 \%$. 
However, it was estimated in the year 1970 that as high as 33\% of the population above 6 years old did not obtained formal education. Therefore we would expect a high proportion of elderly with limited proficiency in the English language. Moreover, $29 \%$ of the population is concentrated in the rural area. And the percentage of people who are able to speak English is limited.

Up to date, the researches in dementia are limited and studies conducted are mainly focus in universities targeting the urban population. This is partly due to the limited validated tools that can be applied to our population. Locally, the only validated cognitive assessment tool is the Mini Mental State Examination. Therefore, the aim of this study is to validate the Malay version of Montreal Cognitive Assessment for cognitively impaired patient. This initial effort also provides researchers with locally validated tool that will lead to further studies into the field of cognitive impairment in this country.

The original English version of Montreal Cognitive Assessment (MoCA) is developed by Dr. Ziad Nasreddine from Montreal, Canada. It was validated and published in 2005 and it showed higher sensitivity than MMSE in detecting mild cognitive impairment. [3]

\section{Methodology}

\subsection{Translation of the Montreal Cognitive Assessment}

A psychogeriatrician has translated the original version of the MoCA into Malay language. This Malay version, designated as MMoCA, was back translated by a bilingual physician, who was blind to the original version. The back translated version was revised by a committee consisting of geriatrician, neurologist, psychogeriatrician, clinical psychologist, and general physicians to fine tune the translation and ascertain the face validity of the MMoCA with regards to linguistic and semantic equivalents.

The instruction for administration of MoCA was also translated into Malay. A pilot study involved 20 Malay-speaking elderly was conducted in Hospital Seberang Jaya (single centre).

The final version of the MMoCA and the MMoCA administration instruction was developed after the pilot study. Investigators and study coordinators have been trained to ensure standardization in the administration of the study tool prior to subject recruitment.

\subsection{Participants}

All subjects are 60 years old and above, consists of 2 groups:

a) Patients with Mild Cognitive Impairment and Alzheimer's Disease

b) Normal Control (NC)

Recruitment took place in 4 government hospitals as listed below:

1. Memory Clinic of Hospital Pulau Pinang

2. Neurology Clinic of Hospital Seberang Jaya
3. Memory Clinic of Hospital Taiping

4. Psychiatric Clinic of Hospital Bukit Mertajam

\subsubsection{Inclusion Criteria}

a) Patients with Mild Cognitive Impairment and Alzheimer's Disease

- $\quad$ Patient above 60 years old diagnosed with Alzheimer's Disease or mild cognitive impairment based on the DSM-IV-TR (American Psychiatric Association 1994), and Alzheimer's Disease based on NINCDS-ADRDA criteria (National Institute of Neurological and Communicative Diseases and Stroke-Alzheimer's Disease and Related Disorders Association). [4]

- A Clinical Dementia Score (CDR) of 0.5 and above. [5]

- Able to speak, read and write Malay language

b) Normal Control

- $\quad$ Elderly above 60 years old

- Able to speak, read and write Malay language

- Independent in activities of daily living

- No past history of psychiatric or neurological disorder

- No memory complaint.

\subsubsection{Exclusion Criteria}

a) Patients with Mild Cognitive Impairment and Alzheimer's Disease

- $\quad$ Patient whom unable to speak, read and write Malay language.

- presence of psychiatric diagnosis (based on DSM-IV-TR) other than dementia.

- $\quad$ Patient with no accompanying caregiver to give inform consent

- $\quad$ Patient with severe somatic and psychiatric symptoms - patients who are restless, aggressive, medically and physically too ill that might significantly impair their cooperation in the study.

b) Normal Control

- $\quad$ Elderly whom unable to speak, read and write Malay language.

- $\quad$ Presence of subjective memory complaint

- $\quad$ Presence of psychiatric illness or physical impairments that would interfere with their ability to complete assessment tools in the study.

\subsection{Sampling Methods}

Convenient sampling was used for this study. Patients presented to memory or neurology clinic were asked to participate in the study. These clinics accept referral for evaluation of elderly with subjective memory complaint, either self-reported or reported by reliable informant. (self-declared and/or complaint from family members).

Normal control group is selected randomly from healthy subjects who attended health screening program in Hospital Seberang Jaya and Hospital Taiping.

\subsection{Instruments and Assessment Tools}

The instruments and assessment tools that was used in this study are as below: 


\subsubsection{Sociodemographic Data for Patients and Elderly From Control Group}

Sociodemographic data was collected for age group, gender, ethnicity, education level, occupation, and household income per month.

\subsubsection{Montreal Cognitive Assessment (MoCA)}

The original English version of MoCA is a tool designed to screen for MCI [2]. It scores from 0 to 30 where the higher scores indicate better cognition. Total score below 26 indicates cognitive impairment. To correct for education effects, 1 point is added for participants with less than or equal to12 years of education.

\subsubsection{Mini-Mental Status Examination (MMSE) MalayVersion}

MMSE is a tool designed to screen for cognitive impairment [6]. It scores from 0 to 30 where the higher scores indicate better cognition. The Malay version of MMSE has been locally validated and modifications done on the repetition question as well as the test of attention [7].

\subsubsection{Clinical Dementia Rating (CDR)}

The CDR is a clinical rating scale developed to rate the severity of dementia [5]. It tests 6 performance areas: memory, orientation, judgment, problem-solving, community affairs, home and hobbies, and personal care. In each area there is a 5 point scale where 0 represents the absence of dementia, 0.5 for questionable, 1 for mild, 2 for moderate, and 3 for severe dementia. For the staging of severity of cognitive impairment, a global CDR score will be applied . A CDR of 0 indicates no cognitive impairment; CDR 0.5 can indicate MCI; CDRs 1, 2 and 3 indicate mild, moderate, and severe dementia, respectively.

\subsection{Tools Administration}

Investigators reviewed the patients' diagnosis as stated in their clinic medical records. Patients who fulfilled the inclusion criteria were invited to participate in the study.

Patients who have given consent to participate in the study were asked to complete the MMoCA and MMSE-Malay administered by the investigator or a trained study team member. All the tests were completed on the same day with 10 minutes break in between the tests.

All patients were asked to return for a second assessment. During the second assessment, assessment was done by a geriatrician, a psychiatrist or a neurologist on the following: i. Detailed structured interview.

ii. Neurological, medical and psychiatric examination.

iii. Severity of dementia assessed using CDR by trained personnel (completed the online Washington University training module for CDR assessment).

The assessors for the second assessment were blinded from the subjects' MMoCA and MMSE scores.

\subsection{Statistical Analysis}

SPSS (version 20.0; SPSS, Inc., Chicago, Ill., USA) was used for all statistical analysis of the following was done:

i. Content validity using Spearman's correlation coefficients between MMoCA scores and those of the MMSE (Malay version) and CDR.

ii. Sensitivity and specificity of the MMoCA for prediction of cognitive impairment and comparison with MMSE

iii. Area under the curve (AUC) in the receiver-operator curves (ROC) to determine predictive value of MMoCA

\section{Results and Discussion}

\subsection{Demographic Data}

Total number of 66 subjects was enrolled in the study, 44 subjects were normal control, 14 were Alzheimer Disease patients and 8 were patients with Mild Cognitive Impairment. The mean age is 66 years old for normal control group, 73.9 years old for Alzheimer's Disease group and 69 years old for Mild Cognitive Impairment group. The mean age for normal control group is significantly younger than the Alzheimer's Disease group and Mild Cognitive Impairment group, $p$-value is less than 0.05 . The rest of the variables are not significantly different in this study.

Gender distribution is quite equal among the studied subjects, $50.8 \%$ are male and $49.2 \%$ are female. The ethnicity distribution is quite similar as Malaysians' composition where majority are Malay, followed by Chinese and Indians.

All the subjects had received formal education, $21.5 \%$ only studied up to primary school level, half of them (49.2\%) completed secondary school, $29.2 \%$ graduated from tertiary education centers or universities. This data does not reflect the real situation in Malaysia, as majority of the subjects who participated in the study are from urban area, whereas the illiterate group is mainly concentrated at rural area, and we believed that those illiterate who stay in urban area rarely come forward to seek treatment because they have poor health awareness about dementia.

In terms of occupation, majority of the Alzheimer's Disease and Mild Cognitive Impairment patients are from professional group, consists of $53.8 \%$ and $83.3 \%$ respectively. This reflects that Alzheimer's Disease and Mild Cognitive Impairment might be under detected for non-professional population in Malaysia. This is may be because of professional group of patients are dealing with more complex daily tasks, where mild cognitive impairment is significant enough to impair their daily lives until they need to seek medical attention.

Table 1. Demographic Data.

\begin{tabular}{ll}
\hline & $\mathbf{n}(\mathbf{\%})$ \\
\hline Diagnosis, N=66 & \\
Normal control & $44(66.7)$ \\
Alzehimer's disease & $14(21.2)$ \\
MCI & $8(12.1)$ \\
Gender & \\
\hline
\end{tabular}




\begin{tabular}{ll}
\hline & $\mathbf{n}(\mathbf{\%})$ \\
\hline Male & $33(50.8)$ \\
Female & $32(49.2)$ \\
Ethnicity & $33(50.8)$ \\
Malay & $24(36.9)$ \\
Chinese & $8(12.3)$ \\
Indian & \\
Education level & $14(21.5)$ \\
Primary & $32(49.2)$ \\
Secondary & $19(29.2)$ \\
Tertiary \& University & \\
None & \\
Marital status & $8(12.5)$ \\
Single & $42(65.6)$ \\
Married & $1(1.6)$ \\
Separated & $2(3.1)$ \\
Divorced & $11(17.2)$ \\
Widowed & \\
Occupation & $2(3.9)$ \\
Legislator & $3(5.9)$ \\
Operator & $6(11.8)$ \\
Technician & $21(41.2)$ \\
Professional & $7(13.7)$ \\
Housewife & $4(7.8)$ \\
Service & $6(11.8)$ \\
Clerical & $2(3.9)$ \\
Skilled worker & $3(4)$. \\
Household income (RM) & $23(35.9)$ \\
Less than 500 & $12(18.8)$ \\
500-1499 & $13(20.3)$ \\
1500-2499 & $4(6.3)$ \\
2500-3499 & $9(14.1)$ \\
3500-4499 & \\
Above 4500 & \\
\hline & \\
& \\
\hline
\end{tabular}

Table 2. Demographic Data according to normal control group, Alzheimer's Disease group and Mild Cognitive Impairment group.

\begin{tabular}{|c|c|c|c|}
\hline & NC n=44 (\%) & $\mathrm{AD} n=14(\%)$ & MCI $n=8(\%)$ \\
\hline Age & 66.0 & 73.9 & 69.0 \\
\hline \multicolumn{4}{|l|}{ Gender } \\
\hline Male & $21(48.8)$ & $6(42.9)$ & $6(75.0)$ \\
\hline Female & $22(51.2)$ & $8(57.1)$ & $2(25.0)$ \\
\hline \multicolumn{4}{|l|}{ Ethnicity } \\
\hline Malay & $25(58.1)$ & $6(42.9)$ & $2(25.0)$ \\
\hline Chinese & 15 (32.6) & $6(42.9)$ & $4(50.0)$ \\
\hline Indian & $4(9.3)$ & $2(14.2)$ & $2(25.0)$ \\
\hline \multicolumn{4}{|l|}{ Education level } \\
\hline Primary & $10(23.3)$ & $4(28.6)$ & $0(0)$ \\
\hline Secondary & $25(58.1)$ & $4(28.6)$ & $3(37.5)$ \\
\hline Tertiary & $8(18.6)$ & $6(42.9)$ & $5(62.5)$ \\
\hline None & $0(0)$ & $0(0)$ & $0(0)$ \\
\hline \multicolumn{4}{|l|}{ Marital status } \\
\hline Single & $7(16.7)$ & $0(0)$ & $1(12.5)$ \\
\hline Married & $27(64.3)$ & $8(57.1)$ & $7(87.5)$ \\
\hline Separated & $1(2.4)$ & $0(0)$ & $0(0)$ \\
\hline Divorced & $1(2.4)$ & $1(7.1)$ & $0(0)$ \\
\hline Widowed & $6(14.3)$ & $5(35.7)$ & $0(0)$ \\
\hline \multicolumn{4}{|l|}{ Occupation } \\
\hline Legislator & $1(3.1)$ & $1(7.7)$ & $0(0)$ \\
\hline Operator & $1(3.1)$ & $2(15.4)$ & $0(0)$ \\
\hline Technician & $5(15.5)$ & $1(7.7)$ & $0(0)$ \\
\hline Professional & $9(28.1)$ & $7(53.8)$ & $5(83.8)$ \\
\hline Housewife & $5(15.5)$ & $2(15.4)$ & $0(0)$ \\
\hline Service & $4(12.6)$ & $0(0)$ & $0(0)$ \\
\hline Clerical & $6(18.8)$ & $0(0)$ & $0(0)$ \\
\hline $\begin{array}{l}\text { Skilled worker } \\
\text { Income (RM) }\end{array}$ & $1(3.1)$ & $0(0)$ & $1(16.7)$ \\
\hline
\end{tabular}

\begin{tabular}{llll}
\hline & NC $\mathbf{n = 4 4}(\mathbf{\%})$ & AD $\mathbf{n}=\mathbf{1 4}(\%)$ & MCI $\mathbf{n}=\mathbf{8}(\mathbf{\%})$ \\
\hline$<500$ & $3(7.1)$ & $0(0)$ & $0(0)$ \\
$500-1499$ & $17(40.5)$ & $3(21.4)$ & $3(37.5)$ \\
$1500-2499$ & $8(19.0)$ & $3(21.4)$ & $1(12.5)$ \\
$2500-3499$ & $6(14.3)$ & $5(35.7)$ & $2(25.0)$ \\
$3500-4499$ & $3(7.1)$ & $1(7.1)$ & $0(0)$ \\
$>4500$ & $5(11.9)$ & $2(14.3)$ & $2(25.0)$ \\
\hline
\end{tabular}

\subsection{Receiver-operator Curves (ROC)}

For ROC analysis, significant results only can be obtained by using MMoCA to differentiate $\mathrm{CDR} 0$ from $\mathrm{CDR}>0$ (i.e. grouping CDR 0.5 and CDR 1). We cannot differentiate CDR 0 from CDR 0.5 due to small sample size in Mild Cognitive Impairment group.

Area under the curve for MMOCA is better than MMSE-Malay in differentiating CDR 0 from CDR $>0$. With the cut off point of less than 22, MMoCA has the sensitivity of 0.824 and specificity of 0.818 to detect cognitive impairment. Whereas MMSE-Malay only has sensitivity of 0.765 and specificity of 0.636 at the cut off point of less than 27 .

Table 3. Sensitivity and Specificity of MMoCA \& MMSE-Malay.

\begin{tabular}{cccc}
\hline & Cut off point & Sensitivity & Specificity \\
\hline MMoCA & 22 & 0.824 & 0.818 \\
MMSE-Malay & 27 & 0.765 & 0.636 \\
\hline
\end{tabular}

\subsection{Limitation}

The major limitation in our study is the number of patients with Mild Cognitive Impairment is very few in all 4 recruitment centers. After data collection, we only managed to analyze the ROC for patients with CDR $0.5-1$. We do not have enough number of patients to do ROC analysis for CDR 0.5 only (MCI). This is may be due to lack of awareness among the public and health care professional in detecting mild cognitive impairment, and some of them might be missed by using MMSE as an assessment tool. Therefore we are looking forward to make more accurate and early diagnosis for those patients suffered from mild cognitive impairment after this MMoCA test has validated.

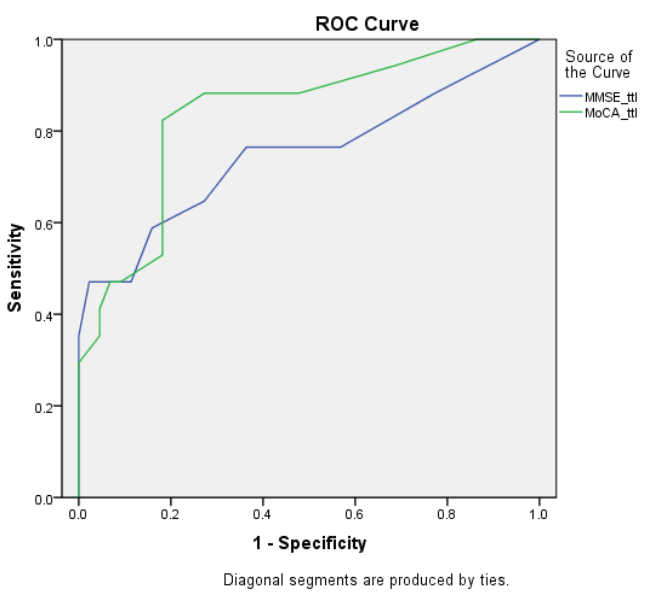

Figure 1. Receiver-operator Curve (ROC) 
Table 4. Area under curve.

\begin{tabular}{|c|c|c|c|c|c|}
\hline \multirow{2}{*}{ Test Result Variable(s) } & \multirow{2}{*}{ Area } & \multirow{2}{*}{ Std. Error ${ }^{a}$} & \multirow{2}{*}{ Asymptotic Sig. } & \multicolumn{2}{|c|}{ Asymptotic $95 \%$ Confidence Interval } \\
\hline & & & & Lower Bound & Upper Bound \\
\hline MMSE_ttl & .749 & .081 & .003 & .591 & .908 \\
\hline $\mathrm{MoCA}{ }^{-} \mathrm{ttl}$ & .838 & .059 & .000 & .723 & .954 \\
\hline
\end{tabular}

The test result variable(s): MMSE_ttl, MoCA_ttl has at least one tie between the positive actual state group and the negative actual state group. Statistics may be biased.

a. Under the nonparametric assumption

b. Null hypothesis: true area $=0.5$

\section{Conclusion}

This study showed that the translated Malay version of Montreal Cognitive Assessment is a valid, reliable, and clinically useful screening tool for patients with cognitive impairment in Malaysia population.

\section{Acknowledgement}

The authors would like to express our gratitude to all the doctors and staffs involved in the study.

\section{References}

[1] Asia Pacific Members of Alzheimer's Disease International, Dementia in the Asia Pacific region: The epidermic is here, executive summary of a report by Access economy pty limited, 2006.

[2] Department of Statistics Malaysia, Population distribution and basic demographic characteristic report 2010.
[3] Ziad S. Nasreddine, Natalie A. Phillips, Valerie Bedirian, Simon Charbonneau, Victor Whitehead, Isabelle Collin et al, The Montreal cognitive assessment, MOCA: A brief screening tool for mild cognitive impairment, Journal of America Geriatric Society, 2005;53(4): 695-99

[4] McKhann G, Drachman D, Folstein M, Katzman R, Price D, Stadlan EM, Clinical diagnosis of Alzheimer's disease: Report of the NINCDS-ADRDA workgroup under the auspices of department of health and human services task force on Alzheimer's disease, Journal of Neurology, 1984; 34(7): 939-44.

[5] Morris JC, The Clinical Dementia Rating (CDR) : Current version and scoring rules, Journal of Neurology, 1993; 43(11): 2412-14

[6] Folstein MF, Folstein SE, McHugh PR, Mini Mental State: A practical method for grading the cognitive state of patients for the clinician, Journal of Psychiatric Research, 1975; 12(3): 189-98

[7] Zarina ZA, Zahiruddin $\mathrm{O}$, Che Wan $\mathrm{AH}$, Validation of Malay mini mental state examination, Malaysia Journal of Psychiatry, 2007; 16(1): 16-19. 